\title{
Dietary Cholesterol Protects Anesthesia-Induced Cognitive Deficits in Wistar Rats
}

\author{
Shibiao Chen ${ }^{1}$, Weicheng Liu ${ }^{1}$, Yanping Zhan ${ }^{1}$, Ranran $\mathrm{Xu}^{1}$, Zhang $\mathrm{Hao}^{1}$ and \\ Jichun Liu ${ }^{2 *}$ \\ ${ }^{1}$ Department of Anesthesiology, ${ }^{2}$ Department of Cardiothoracic Surgery, The First Affiliated Hospital of \\ Nanchang University, Nanchang City, Jiangxi Province 330006, China
}

*For correspondence: Email: liujichun999@gmail.com; Tel/Fax: 0086-971-88692707

Received: 12 January 2015

Revised accepted: 18 May 2015

\begin{abstract}
Purpose: To evaluate the effect of cholesterol on frequent exposure of anesthesia-induced cognitive impairment in wistar rats.

Methods: Healthy wistar rats were divided in two groups, the gp I rats fed with regular diet and gp II with cholesterol diet. These groups were further divided into sub-groups as gp la $(n=8)$ and gp lla $(n=8)$. These sub-groups received weekly exposure of anesthesia for 6 weeks. Animals were anesthetized by subcutaneous sodium thiopental injection. Cortical nerve growth factor levels were measured by indirect sandwich enzyme-linked immunosorbent assay (ELISA) while total protein was determined by Bradford protein assay.

Results: Group Ila (cholesterol-fed animals) as well as Group IIb (cholesterol-fed followed by anesthesia) showed significant increase in body weight (25 to $50 \mathrm{~g}, p<0.03$ ), but no such increase was observed in other groups. However, group Ib showed a significant $(43.07 \%, p<0.001)$ decrease in the level of nerve growth factor when compared with group la. Moreover, significantly decreased cytokines IL-1 $\beta$ levels (59.09\%, $p<0.005)$ and TNF- $\alpha(20 \%, p<0.025)$ of group Ila more effectively than in group la rats. Microglial marker showed significantly increase $(16.66 \%, p<0.025)$ in cholesterol diet group. Overall increase in leakage of anti-rat lgG (blood brain barrier marker) was found in both groups (IIa and IIb).

Conclusion: The results suggest that dietary cholesterol protects or neutralizes anesthesia-induced cognitive deficits in rats.
\end{abstract}

Keywords: Cognitive deficit, Cholesterol diet, Blood-brain barrier, Nerve growth factor, Inflammation marker, Microglial marker, Cytokines

Tropical Journal of Pharmaceutical Research is indexed by Science Citation Index (SciSearch), Scopus, International Pharmaceutical Abstract, Chemical Abstracts, Embase, Index Copernicus, EBSCO, African Index Medicus, JournalSeek, Journal Citation Reports/Science Edition, Directory of Open Access Journals (DOAJ), African Journal Online, Bioline International, Open-J-Gate and Pharmacy Abstracts

\section{INTRODUCTION}

Exposure to general anesthesia causes widespread apoptotic neurodegeneration in the developing mammalian brain [1]. Some researchers suggested an association between early exposure to anesthesia and long-term impairment of cognitive development [2,3]. Anesthesia-induced cognitive impairment may depend on developmental stage, anesthetic agent, and number of exposures. Cholesterol is a critical component of the central nervous system involved in the maintenance of normal brain function including synapse formation, receptor function, synaptic plasticity, and signaling [4]. Cholesterol diet reverses the problems that are associated with learning and memory in rats which are either deficient in cholesterol or deficit 
in cholesterol synthesis [5]. Converging evidence indicates that altered cholesterol levels and/or metabolism are associated with changes in learning and memory [6]. There are relatively few studies on memory retention following cholesterol diet. However, a recent investigation indicates that dietary cholesterol may retard longterm memory [7]. In addition to changes in learning and memory, studies have also shown that cholesterol can impact brain pathology, including breakdown of blood brain barrier (BBB), microglia activation, apoptosis, elevated inflammatory marker expression, myelin sheath and axon damage, $\beta$-amyloid accumulation, tau phosphorylation, and cerebrovascular changes [7-9]. Based on the persuasive evidence for the beneficial effects of cholesterol against cognitive dysfunctions, the present study was conducted to examine the therapeutic effects of cholesterolrich diet on cognitive deficits or brain pathology in rat model.

\section{EXPERIMENTAL}

\section{Animal grouping and cholesterol diet}

The twelve-week-old healthy Wistar rats were divided into two groups the group I $(n=16)$ rats fed with regular/normal diet and group II $(n=16)$ with cholesterol diet for 2 months respectively. These groups were further divided into subgroups as group la $(n=8)$ and group Ila $(n=8)$ for received weekly exposure of anesthesia for 6 weeks, respectively.

The cholesterol diet consisted (50 $\mathrm{g}$ of cholesterol, $250 \mathrm{~g}$ sodium cholate, $135 \mathrm{~g}$ of casein, $150 \mathrm{~g}$ of maltodextrin, $100 \mathrm{~g}$ of sucrose, $40 \mathrm{~g}$ of soybean oil, $50 \mathrm{~g}$ of fiber, $40 \mathrm{~g}$ of mixed mineral, $2 \mathrm{~g}$ of L-cystine, $1.4 \mathrm{~g}$ of choline chloride, $0.001 \mathrm{~g}$ of butyl-hydroxytoluol, $0.002 \mathrm{~g}$ of folic acid and $1 \mathrm{~g}$ of chocolate aroma) per $\mathrm{Kg}$ body weight of animals.

The animals were maintained in accordance with the U.S. National Institutes of Health Guide for the Care and Use of Laboratory Animals (NIH Publication 80-23, revised 1996) and all procedures were approved by the Ethical Committee of the First Affiliated Hospital of Nanchang University (approval ref. no IECA/FAHNU/2011-021) [10, 12]. After three months, animals on the normal diet $(n=10)$ and the cholesterol diet $(n=10)$ were given weekly i.e. injections containing $100 \mu \mathrm{L}$ of heparinized saline for two months via the dorsal penile vein. Prior to injection, each rat was anaesthetized by an intraperitoneal injection of thiopental (400 $\mu \mathrm{l} / 100 \mathrm{~g}, 12.5 \mathrm{mg} / \mathrm{mL})$. Animals receiving a three-month normal diet $(n=7)$ or cholesterol diet $(n=6)$ undisrupted by anesthesia treatments served as control animals.

\section{Sample preparation}

Animals were anesthetized by subcutaneous sodium thiopental $(12.5 \mathrm{mg} / \mathrm{ml}, 1 \mathrm{~mL})$ injection. Brains were removed and the frontal cortices were dissected from the left hemisphere. One section of cortex was frozen at $-80{ }^{\circ} \mathrm{C}$. The right brain hemisphere was post-fixed overnight in $4 \%$ paraformaldehyde (PFA) and then stored in $20 \%$ sucrose/sodium azide solution. The frozen cortical tissue for ELISA evaluation was dissolved in $100 \mu \mathrm{L}$ ice-cold PBS containing protease inhibitor cocktail (P-8340, Sigma), homogenized using an ultrasonic device (Hielscher Ultrasonic Processor, Germany) and then centrifuged at $16,000 \mathrm{~g}$ for $10 \mathrm{~min}$ at $4{ }^{\circ} \mathrm{C}$. The supernatant was collected and samples were stored at $-80{ }^{\circ} \mathrm{C}$ until further use. Total protein was determined by Bradford protein assay.

\section{Measurement of cortical nerve growth factor (NGF)}

Cortical NGF levels were measured in cortex extracts using an indirect sandwich enzymelinked immunosorbent assay (ELISA) as previously described [11]. The 96-well plates were coated with a monoclonal anti-NGF antibody diluted in carbonate coating buffer $(\mathrm{pH}$ 9.7) and incubated overnight at $4{ }^{\circ} \mathrm{C}$. The plates were then blocked using $1 \mathrm{x}$ blocking buffer (200 $\mu \mathrm{l} /$ well) for $1 \mathrm{~h}$ at $20{ }^{\circ} \mathrm{C}$. Following incubation, NGF standards or tissue extracts were added to the plates and incubated for $6 \mathrm{~h}$ at $20{ }^{\circ} \mathrm{C}$. After washes, the plates were incubated with a monoclonal rat anti-NGF antibody overnight at 4 ${ }^{\circ} \mathrm{C}$. After a second round of washings, the plates were incubated with horse radish peroxidaseconjugated anti-rat antibody (1:4000) for $2 \mathrm{~h}$ at $20{ }^{\circ} \mathrm{C}$. Plates were again washed and incubated with enzyme substrate (TMB One solution, Promega) for $15 \mathrm{~min}$ at $20{ }^{\circ} \mathrm{C}$. The enzyme reaction was stopped by adding $1 \mathrm{~N} \mathrm{HCl}$ and the absorbance was measured at $450 \mathrm{~nm}$ by a microplate ELISA reader. Sample values were calculated from a standard curve in the linear range. The assay detection limit was $10 \mathrm{pg} / \mathrm{mL}$ NGF.

\section{Evaluation of inflammatory proteins}

The detection of inflammatory proteins such as monocyte chemotactic protein-1 (MCP-1), macrophage inflammatory protein-2 (MIP-2), tumor necrosis factor- $\alpha$ (TNF- $\alpha$ ) and interleukin$1 \beta$ (IL-1 $\beta$ ) was performed using the Thermo 
Scientific Search Light Protein Array Technology (THP Medical Products, Vienna) according to the manufacture's recommendations (Bio-Rad) and as described previously [12]. Cell extracts or calibrated standards were added to coated wells of the provided plate and incubated for $3 \mathrm{~h}$. After washing, the biotinylated antibodies were added and following $30 \mathrm{~min}$ incubation the wells were washed again and incubated with streptavidinhorseradish peroxidase conjugate. After the final washing step the Super Signal Chemiluminescent substrate was added. All incubation steps were carried out on a shaker at $20{ }^{\circ} \mathrm{C}$. The luminescent signal was detected using a compatible CCD imaging and analysis system and the absorbance was measured at $450 \mathrm{~nm}$. The concentration of each sample was quantified by comparing the spot intensities with the corresponding standard curves calculated from the standard sample results using the Search Light Array Analyst Software. Integrated density values were proportional to the concentrations of bound proteins. Standard curves, raw data and final $\mathrm{pg} / \mathrm{mL}$ concentrations for each analyte and each sample were reviewed in the array software and exported to Microsoft Excel Software for further statistical analysis. Sample values were calculated from a standard curve in the linear range.

\section{Statistical analysis}

One-Way ANOVA was used for the analysis of statistical significance in SPSS version 11
(Chicago IL.) for cognitive performance and all other measures. All data are reported as mean \pm SEM $(n=$ independent experiments or individual animals). Level of significance was performed between $p<0.05$ or $p<0.0001$ for all statistical tests.

\section{RESULTS}

The body weight of groups la (normal diet animals) and $\mathrm{lb}$ (normal diet followed by anesthesia) was 320 to $350 \mathrm{~g}$, and for groups Ila (cholesterol diet animals) and Ilb (cholesterol diet + anesthesia), it was between 375 and $400 \mathrm{~g}$. Thus, approximately 25 to $50 \mathrm{~g}$ increase $(p<$ 0.03 ) in body weight for groups Ila and IIb was observed. However, there is no significant variation in body weight between group la $\& \mathrm{lb}$ as well as between group Ila \& IIb. Thus anesthesia showed no effect on body weight of treated animals.

However, group Ilb (cholesterol+anesthesia) animals exhibited significantly $\quad(47.05 \%, p<$ 0.05 ) elevated levels of cortical NGF when compared with group Ila, indicating that exposure to anesthesia might lead to the dysfunction of cholinergic system under normal die (Fig 1). Group IIb animals also exhibited significantly $(p<$ $0.0001)$ elevated levels of cortical NGF compared to that of group lb animals (Fig 1).

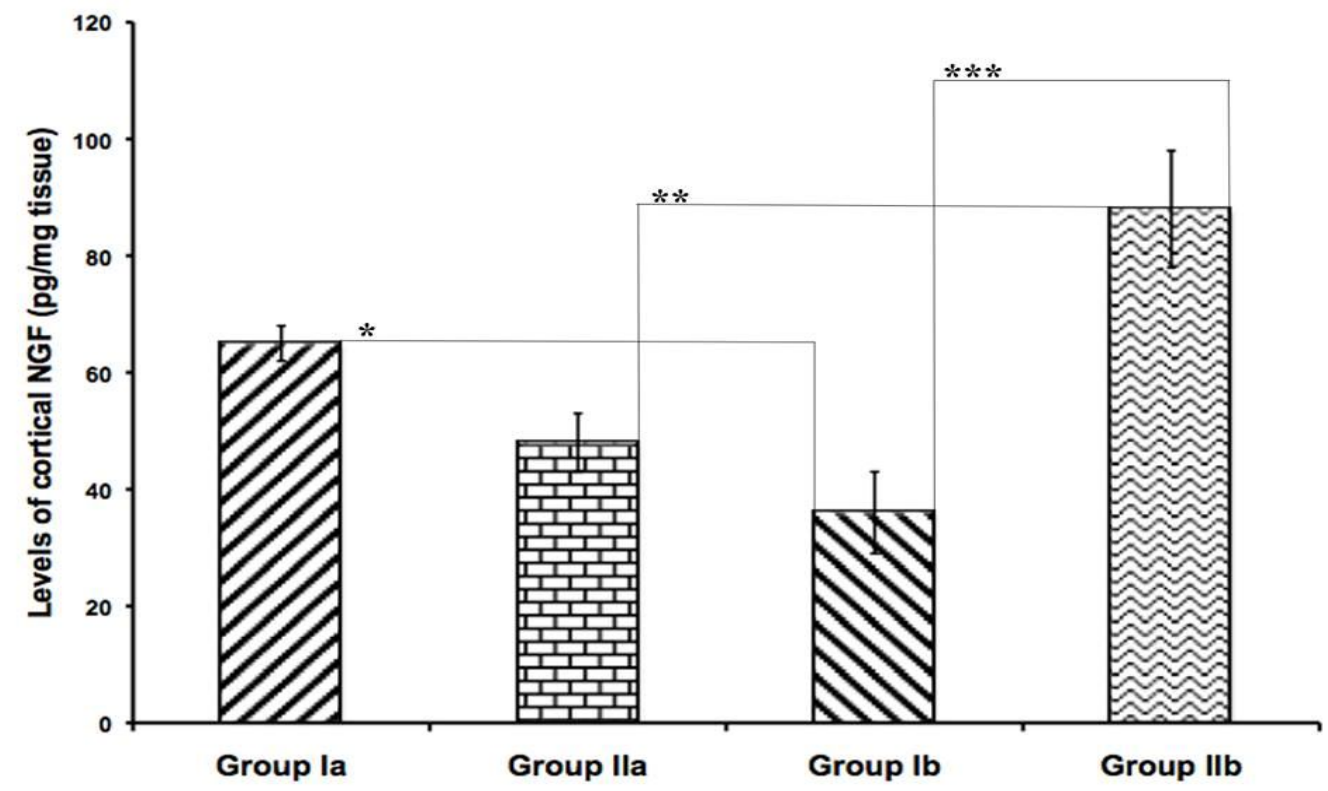

Figure 1: Cholesterol diet protects anesthesia-induced impairment on the cortical nerve growth factor (NGF), which is associated with cholinergic neuron survival and function. The asterisks represent the significant difference between groups with respective $p$ values such as ${ }^{*} p<0.001$; ${ }^{* *} p<0.05$; ${ }^{* * *} p<0.0001$ 
Moreover, significantly decreased levels of cytokines IL-1 $\beta(59.09 \%, p<0.005)$ and TNF- $\alpha$ (20\%, $p<0.025)$ observed in group lla (cholesterol fed) rats than that of group la (normal diet) rats. However, no significant difference in the levels of MCP-1 and MIP2 was observed in group la and group Ila (Figs 2 and 3 ). The microglial marker was assessed by OX42 staining, which showed significantly increase
$(16.66 \%, p<0.025)$ effect on cholesterol diet or group lla than that of group la (normal diet). However, no significant difference was found between group $\mathrm{lb}$ and group Ilb rats (Figs 2 and 3 ) we observed the immuno reactivity of anti-rat IgG staining where we found significantly increased $(49.80 \%, p<0.05)$ staining pattern in group II rats than that of group I rats (Fig 4).

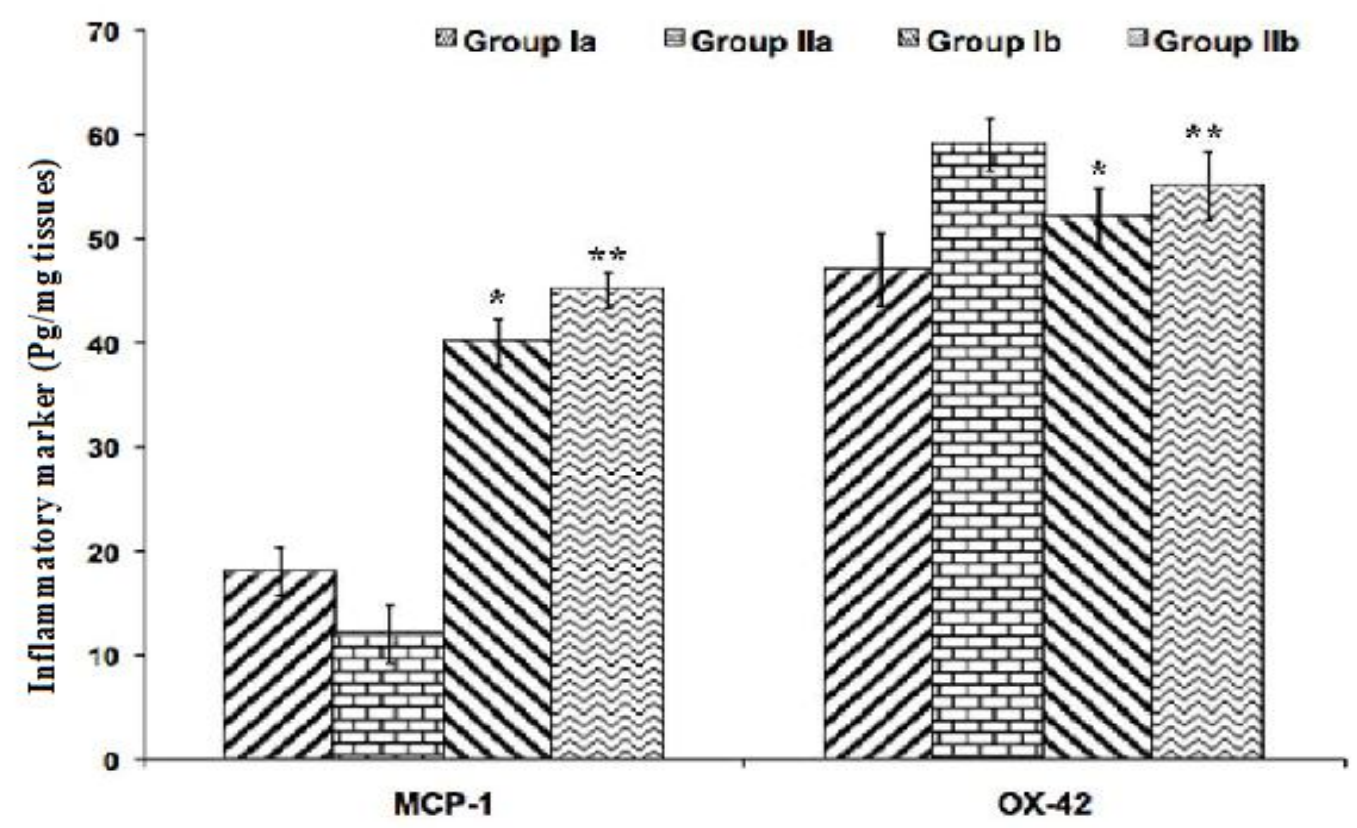

Figure 2: The effects of diet and anesthesia exposure on cortical inflammatory markers MCP-1 and OX-42. The asterisks represent the significant difference between groups with respective $p$ values such as ${ }^{*} p<0.005$; ${ }^{* *} p<$ 0.025

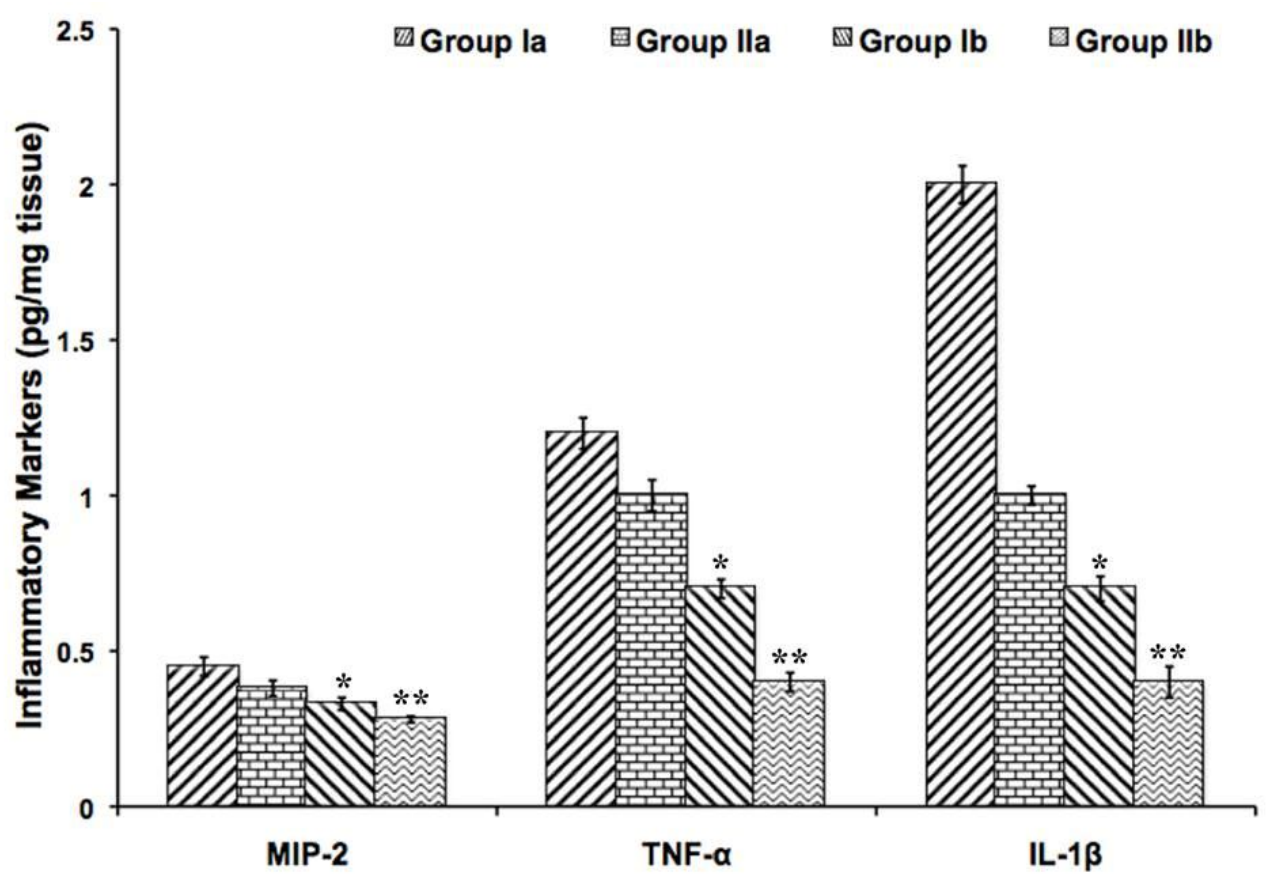

Figure 3: The effects of diet and anesthesia exposure on cortical inflammatory markers MIP2, TNF- $\alpha$, IL-1 $\beta$. The asterisks represent the significant difference between groups with respective $p$ values such as ${ }^{*} p<0.005$; ${ }^{\star *} p<$ 0.025 


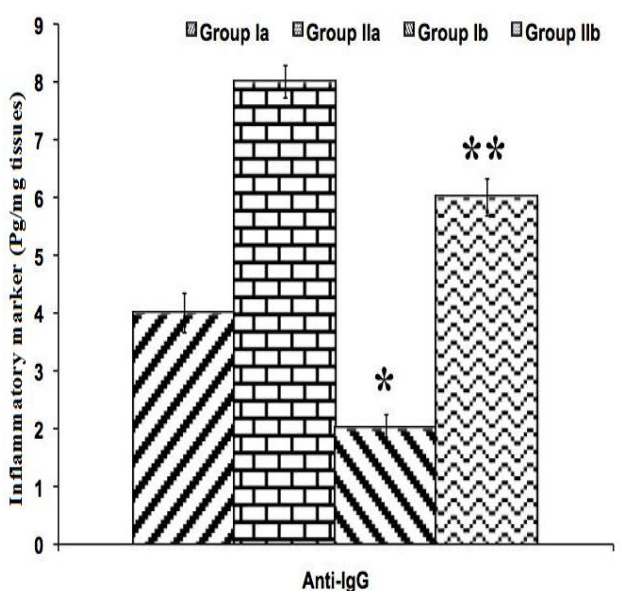

Figure 4; The effects of diet and anesthesia exposure on cortical inflammatory marker Anti rat IgG. The asterisks represent the significant difference between groups with respective $p$ values such as * $p<0.005$; ** $p<0.025$

\section{DISCUSSION}

In this study, we have attempted to show the beneficial aspect of cholesterol in protecting against the deficits in spatial learning and impairment in nerve growth factors. However, some researchers have proposed the impairment of cognitive function in humans as a cause of repeated exposure of anesthesia [2,3]. In addition to changes in learning and memory, some reports revealed the impact of cholesterol on brain pathology like BBB breakdown, microglia activation, elevated inflammatory marker expression, myelin sheath and axon damage, $\quad \beta$-amyloid accumulation and cerebrovascular changes $[7,8]$.

It has been reported that diet-induced hypercholesterolemia, or high cholesterol, in male Sprague Dawley rats results in impaired learning and memory, reduced survival of NBM (Nucleus Basalis of Meynert) cholinergic neurons, reduced cortical acetylcholine, elevated inflammatory factors, elevated cortical $\beta$-amyloid, tau, and phospho-tau 181 and increased presence of small cortical BBB (blood brain barrier) feedings [13].

Our study was designed to induce the cognitive deficits via frequent exposure of anesthesia (Thiopental) in wistar rats. The cholesterol diet was administered to protect these deficits and the experimental groups were designed with appropriate control groups determining the levels of nerve growth factor and cortical inflammatory markers.
We observed a minor difference in body weight between cholesterol-fed (group II) and normalfed (group I) animals. Interestingly, there were no significant changes found between group la (normal diet fed animals) \& Ib (normal diet followed by anesthesia) as well as group Ila (cholesterol fed animals) \& Ilb (cholesterol followed by anesthesia). This is consistent with the similar study performed in male Sprague Dawley rats [13].

Earlier investigations have reported the impact of anesthesia towards profound cognitive impairment in learning and memory leading to defects in brain function at various levels [14]. Similarly, we observed significantly decreased level of spatial learning, neural growth factors and alterations of pro-inflammatory markers in rats exposed to anesthesia (thiopental) with normal diet (group lb) than that of non-exposed normal-fed (group la) animals.

Moreover, the vital effect of cholesterol diet in this experiment involves increased microglial and IgG cortical staining, which propose the activation of neuro-inflammation marker microglia and disruption of blood brain barrier

The mechanism of thiopental-induced cognitive impairment is still not clear, however, we believe that the behavioral, cellular, and biochemical deficits in these animals are caused from frequent exposure of anesthesia. This demonstrated that thiopental promotes enhanced GABA release resulting in inhibitory synaptic transmission [15].

Our investigation on Cortical NGF also revealed a significantly enhancement in the levels of NGF in group Ilb when compared with group Ib. This study is in line with other studies that cholesterol can modulate anesthetic suppression of ion channel currents [16].

The frequent exposure of anesthesia-induced disruption of membrane signal transduction might be protected by cholesterol diet, which facilitate in maintaining cholesterol homeostasis [17]. Nevertheless, the rationale is to consider that dietary cholesterol cannot cross the BBB.

\section{CONCLUSION}

On studying, the therapeutic effects of cholesterol-rich diet on cognitive deficits or brain pathology in rat model. We concluded that repeated exposure of anesthesia induced cognitive dysfunction in treated animals that could be protected by means of cholesterol diet. 


\section{REFERENCES}

1. Rizzi S, Carter LB, Ori C, Jevtovic-Todorovic V. Clinical anesthesia causes permanent damage to the fetal guinea pig brain. Brain Pathol 2008; 18 (2):198-210.

2. Wilder RT, Flick RP, Sprung J, Katusic SK, Barbaresi WJ, Mickelson C, Gleich SJ, Schroeder DR, Weaver $A L$, Warner DO. Early exposure to anesthesia and learning disabilities in a population-based birth cohort. Anesthesiology 2009; 110 (4): 796-804.

3. Kalkman CJ, Peelen L, Moons KG, Veenhuizen M, Bruens M, Sinnema G, de Jong TP. Behavior and development in children and age at the time of first anesthetic exposure. Anesthesiology 2009; 110 (4): 805-812.

4. Hohsfield LA, Ehrlich D, Humpel C. Cholesterol diet counteracts repeated anesthesia/infusion-induced cognitive deficits in male Brown Norway rats. Neurobiol Learn Mem 2013; 106: 154-62.

5. Voikar V, Rauvala H, Ikonen E. Cognitive deficit and development of motor impairment in a mouse model of Niemann-Pick type $C$ disease. Behav Brain Res 2002; 132(1): 1-10.

6. Darwish DS, Wang D, Konat GW, Schreurs BG. Dietary cholesterol impairs memory and memory increases brain cholesterol and sulfatide levels. Behav Neurosci 2010; 124 (1): 115-123.

7. Schreurs $B G$, Wang $D$, Smith-Bell $C A$, Burhans $L B$, Bell $R$, Gonzalez-Joekes J. Dietary cholesterol concentration and duration degrade long-term memory of classical conditioning of the rabbit's nictitating membrane response. Int $\mathrm{J}$ Alzheimers Dis 2012; 7326-7334.

8. Berkman Z, Tanriover G, Acar G, Sati L, Altug T, Demir $R$. Changes in the brain cortex of rabbits on a cholesterol-rich diet following supplementation with a herbal extract of Tribulus terrestris. Histol Histopathol 2009; 24 (6): 683-692.

9. Sharma S, Prasanthi RPJ, Schommer E, Feist G, Ghribi O. Hypercholesterolemia-induced Abeta accumulation in rabbit brain is associated with alteration in IGF1 signaling. Neurobiol Dis 2008; 32 (3): 426-432.

10. Approved protocol from Animal Ethical Committee, the First Affiliated Hospital of Nanchang University for the use of animal experiments, Reference number: 2011021.

11. Böttger D, Ullrich C, Humpel C. Monocytes deliver bioactive nerve growth factor through a brain capillary endothelial cell monolayer in vitro and counteract degeneration of cholinergic neurons. Brain Res 2010; 1312: 108-119.

12. Hohsfield LA, Humpel C. Homocysteine enhances transmigration of rat monocytes through a brain capillary endothelial cell monolayer via ICAM-1. Curr Neurovasc Res 2010; 7(3): 192-200.

13. Ullrich C, Pirchl M, Humpel C. Hypercholesterolemia in rats impairs the cholinergic system and leads to memory deficits. Mol Cell Neurosci 2010; 45: 408417.

14. Wang DS, Orser BA. Inhibition of learning and memory by general anesthetics. Can J Anesth 2011; 58 (4): 167-177.

15. Hirota K, Sasaki R, Yamazaki M. Pre-synaptic function explains age dependent actions of general anesthetics in the rat hippocampal synaptic transmission. Toxicol In Vitro 2012; 26(6): 872-877.

16. Rehberg B, Urban BW, Duch DS. The membrane lipid cholesterol modulates anesthetic actions on a human brain ion channel. Anesthesiology 1995; 82 (3): 749758.

17. Levitan I, Fang Y, Rosenhouse-Dantsker A, Romanenko V. Cholesterol and ion channels. Subcell Biochem 2010; 51: 509-549. 